\title{
LEFT DEFINITE MULTIPARAMETER EIGENVALUE PROBLEMS
}

BY

PAUL BINDING ${ }^{1}$

ABSTRACT. We study the problem

$$
T_{m} x_{m}=\sum_{n=1}^{k} \lambda_{n} V_{m n} x_{m}, \quad 0 \neq x_{m} \in H_{m}, m=1, \ldots, k,
$$

where $T_{m}$ and $V_{m n}$ are selfadjoint linear operators on separable Hilbert spaces $H_{m}$, with $T_{m}$ positive, $T_{m}^{-1}$ compact and $V_{m n}$ bounded. We assume "left definiteness" which involves positivity of certain linear combinations of cofactors in the determinant with $(m, n)$ th entry $\left(x_{m}, V_{m n} x_{m}\right)$.

We establish a spectral theory for (*) that is in some way simpler and more complete than those hitherto available for this case. In particular, we make use of operators $B_{n}=\Delta_{n}^{-1} \Delta_{0}$, where the $\Delta_{n}$ are determinantal operators on $\otimes_{m=1}^{k} H_{m}$. This complements an established approach to the alternative "right definite" problem (where $\Delta_{0}$ is positive) via the operators $\Gamma_{n}=\Delta_{0}^{-1} \Delta_{n}$.

1. Introduction. In this section we shall discuss how the left and right definiteness conditions arose, and what methods and results are currently available for the left definite case. Then in 1.3 we shall summarise our approach here. Throughout, we shall be considering the system (*) of the abstract.

1.1 Definiteness conditions. Early work on (*) was motivated by separation of variables in certain classical boundary value problems. The resulting ordinary differential equations in most cases do satisfy the conditions we impose on the operators $T_{m}$ and $V_{m n}$, the $H_{m}$ being $L_{2}$ spaces. The bulk of the literature (which also covers finite dimensional equations, including difference approximations) involves sign definiteness of the determinant $\delta_{0}(x)$ whose $(m, n)$ th entry is $\left(x_{m}, V_{m n} x_{m}\right)$. Atkinson [1] has treated such problems systematically, and his work, together with various recent extensions, now provides an analogue of much of the basic spectral theory for a single equation

$$
T x=\lambda V x, \quad x \neq 0,
$$

with $(x, V x)$ sign definite. Such problems are nowadays called "right definite".

Actually, various right definiteness conditions have been used, and two which are relevant to our discussion are the strong $\delta_{0}$ condition

$$
\delta_{0}(x) \geqslant \gamma\left\|x_{1}\right\|^{2} \cdots\left\|x_{k}\right\|^{2}
$$

for some $\gamma>0$, and the condition that $\Delta_{0}$ be "strongly positive" $\left(\Delta_{0} \gg 0\right)$ i.e.

$$
\Delta_{0}-\beta I \geqslant 0 \text { on } H
$$

Received by the editors July 16, 1980.

1980 Mathematics Subject Classification. Primary 15A18, 47B25; Secondary 15A69, 47D20.

${ }^{1}$ Research supported by NSERC of Canada. 
for some $\beta>0$. Here $H$ is the Hilbert tensor product $\otimes_{m=1}^{k} H_{m}, I$ is the corresponding identity operator, and $\Delta_{0}$ is formally defined by $\otimes \operatorname{det}\left[V_{m n}\right]$-we shall construct such operators explicitly in $\$ 2$. If

$$
x^{\otimes}=x_{1} \otimes \cdots \otimes x_{k}
$$

then $\left(x^{\otimes}, \Delta_{0} x^{\otimes}\right)=\delta_{0}(x)$, so (1.2) implies (1.1) with $\gamma=\beta$. There is a partial converse-(1.2) with $\beta>0$ implies (1.1) for some $\gamma>0$ [2].

Some cases of separation of variables (e.g. of the wave equation into elliptic coordinates) yield systems $(*)$ for which certain linear combinations of cofactors of $\delta_{0}(x)$, rather than $\delta_{0}(x)$ itself, are sign definite. Motivated by such examples, Källström and Sleeman [10] introduced a "left definiteness" condition for systems of differential equations, equivalent to the following:

$$
\sum_{n=1}^{k} \alpha_{n} \delta_{0 m n}(x)\left\|x_{n}\right\|^{2} \geqslant 2 \delta\left\|x_{1}\right\|^{2} \cdots\left\|x_{k}\right\|^{2}, \quad m=1, \ldots, k,
$$

where $\delta>0$, the $\alpha_{n}$ are real and $\delta_{0 m n}(x)$ is the $(m, n)$ cofactor of $\delta_{0}(x)$. Källström and Sleeman subsequently developed an approach to the abstract formulation (*), the definiteness condition involving (operator) cofactors $\Delta_{0 m n}$ of $\Delta_{0}$-cf. (1.2). Again [2] shows that this is equivalent to (1.4), which is the definiteness condition we shall employ below. The use of the word "left" will become clear in 1.2.

Several other definiteness conditions have been proposed, and we shall briefly comment on some of them. Browne [7] uses (1.2) but with $\beta=0$ and some additional conditions - the relation between this and (1.1) with $\gamma=0$ is not known at present. Atkinson [1, p. 107] uses a condition involving an operator $\Delta$ formally given by

$$
\Delta=\otimes\left|\begin{array}{cccc}
\alpha_{0} & \alpha_{1} & \cdots & \alpha_{k} \\
-T_{1} & V_{11} & \cdots & V_{1 k} \\
\vdots & \vdots & & \vdots \\
-T_{k} & V_{k 1} & \cdots & V_{k k}
\end{array}\right|
$$

- we shall call this " $\Delta$-definiteness". Atkinson's treatment of the finite dimensional case [1, Chapters 6,7$]$ has been partially extended to bounded $T_{m}$ by Källström and Sleeman [12] and to unbounded $T_{m}$ by Sleeman [14]. $\Delta$-definiteness includes both left and right definiteness $\left(\alpha_{1}, \ldots, \alpha_{k}\right.$ being the same as in (1.4)) and in the bounded case can be reduced to a real determinant condition [2]. Whether this fact remains true for Sleeman's problem is not known. More general conditions, guaranteeing rather less spectral theory, have been studied by various authors-cf. Turyn [15].

1.2 Tensor product methods. The approach of Källström and Sleeman [10] to the left definite problem is via separation of variables in reverse: they eliminate all but one of the parameters $\lambda_{n}$, deriving a p.d.e. from the o.d.e. system (*). Abstractly, this involves operators on the tensor product $H$, and this is the setting that we shall adopt.

Let us label the cofactor (operator) of $\alpha_{n}$ in (1.5) by $\Delta_{n}, n=0, \ldots, k$. Then it turns out that the elimination of all except $\lambda_{n}$ from (*) yields

$$
\Delta_{n} x^{\otimes}=\lambda_{n} \Delta_{0} x^{\otimes}
$$


in the notation (1.3), under certain conditions. Such conditions have been investigated in their own right, and for example it is enough if $\Delta_{0} \gg 0$ (1.2). Indeed, in the right definite case with bounded $T_{m}$, Browne [5] replaces (*) by the system

$$
\Gamma_{n} x^{\otimes}=\lambda_{n} x^{\otimes}, \quad \Gamma_{n}=\Delta_{0}^{-1} \Delta_{n}, \quad n=1, \ldots, k .
$$

In the left definite case (1.3), it turns out that our conditions on the $T_{m}$ force

$$
\Sigma=\sum_{n=1}^{k} \alpha_{n} \Delta_{n}
$$

to be strongly positive (1.2) i.e. $\Sigma \gg 0$. Then (1.6) may be replaced by

$$
\Sigma x^{\otimes}=\left(\sum_{n=1}^{k} \alpha_{n} \lambda_{n}\right) \Delta_{0} x^{\otimes},
$$

at least if $\Sigma$ is bounded. The motivation for the terms right and left definite can now be seen from (1.6) and (1.8).

It can be shown that the $\Gamma_{n}(1.7)$ commute in the bounded right definite case, and are selfadjoint in a renormed (but homeomorphic) version of $H$. In this way, Browne [5] gives a spectral theory on the basis of Prugovečki's theory of commuting selfadjoint operators [13, §IV.2]. The system

$$
\Gamma_{n} x^{\otimes}=\lambda_{n} x^{\otimes}, \quad \Gamma_{n}=\Delta^{-1} \Delta_{n}, \quad n=1, \ldots, k,
$$

similarly forms the basis of the analysis of [12] for the bounded $\Delta$-definite case. The results are similar to Browne's, although the renorming now involves the $T_{m}$, making completion necessary even in the left definite case if the $T_{m}$ are unbounded [10].

Right definite systems of o.d.e. have been analysed via different limiting devices by Faierman [9] and Browne [4]. Källström and Sleeman [10] analyse a left definite system of o.d.e. via the p.d.e. (1.8) and an auxiliary right definite system of $k-1$ o.d.e. These authors all use ingenious, but sometimes rather lengthy, arguments to derive real discrete spectra with no finite accumulation, and complete orthonormal eigenvectors in $H$. Attempts have been made to extend the abstract theories for right and $\Delta$-definiteness to unbounded (including differential) operators by Browne [6] and Sleeman [14], respectively. At present, however, these appear to involve technical difficulties (I thank Professors Browne and Sleeman for personal communications about their articles).

1.3 Summary. Our aim is to present an analysis of (*) that is in some ways more direct and more general than currently available, but is within the basic framework described in 1.2. We shall derive a spectral theory, including completeness of the eigenvectors. Most of the analysis will be in $H$ (not renormed) and we shall not need to reconsider the individual equations of $(*)$ (as in [6 and 14]) in order to obtain discreteness of the spectrum.

The basic construction of the determinantal operators we need is carried out in $\S 2$. In $\S 3$ we introduce a transformation enabling $(*)$ to be written as

$$
\Delta_{n} x^{\otimes}=\lambda_{n} \Delta_{0} x^{\otimes}, \quad n=1, \ldots, k,
$$

where each $\Delta_{n} \gg 0$-in (1.8) only one such equation was deduced. We also show how to construct compact operators $B_{n}=\Delta_{n}^{-1} \Delta_{0}$. In $\S 4$ we carry out a limiting 
procedure to extend a result of [11] to unbounded $\Delta_{n}$, and we deduce that the $B_{n}$ commute.

The $B_{n}$ are not selfadjoint (unless we renorm $H$ for each $n$ ) but they are similar to compact selfadjoint (noncommuting) operators $B_{n}^{\prime}$. In $\$ 5$ we use this fact to show that the $B_{n}$ have identical finite dimensional eigenspaces generating a set $S$ of common eigenvectors which is fundamental in certain subspaces $K_{l}$ of $H\left(K_{l}=H\right.$ if $\Delta_{0}$ is $\left.1-1\right)$. In $\$ 6$ we show that $S$ corresponds to the eigenvectors of $(*)$, and we deduce the existence of a real discrete spectrum for $(*)$ with no finite accumulation points. Introduction of the $k$ inner products $[,]_{l}$ given by

$$
[x, y]_{l}=\left[x, \Delta_{l} y\right], \quad l=1, \ldots, k,
$$

permits us to construct complete orthonormal bases for the $k$ corresponding orthogonal complements of $\operatorname{Ker} \Delta_{0}$ out of the eigenvectors for $(*)$.

2. Operators on tensor products. Let $\tau_{m 1} \leqslant \tau_{m 2} \leqslant \ldots$ be the eigenvalues of $T_{m}$, repeated according to multiplicity, and let $t_{m 1}, t_{m 2}, \ldots$ be corresponding eigenvectors forming a complete orthonormal basis of $H_{m}, m=1, \ldots, k$. We consider $H$ as the Hilbert tensor product $\otimes_{n=1}^{k} H_{n}$, so

$$
t_{\mathbf{j}}=t_{1 j_{1}} \otimes \cdots \otimes t_{k j_{k}}, \quad \mathbf{j}=\left(j_{1}, \ldots, j_{k}\right),
$$

form a complete orthonormal basis of $H$ as $\mathbf{j}$ ranges over positive integer $k$-vectors. In particular, we shall employ the notation

$$
x=\Sigma_{\mathrm{j}} \xi_{\mathrm{j}} t_{\mathrm{j}}
$$

for an arbitrary element $x \in H$.

We "induce" $V_{m n}$ into $H$ by first defining, in the notation (1.3),

$$
V_{m n}^{\dagger} x^{\otimes}=x_{1} \otimes \cdots \otimes x_{m-1} \otimes V_{m n} x_{m} \otimes x_{m+1} \otimes \cdots \otimes x_{k}, \quad x_{l} \in H_{l},
$$

and then extending $V_{m n}^{\dagger}$ to $H$ by linearity and continuity. Similarly, we define

$$
T_{m}^{\dagger} t_{\mathrm{j}}=\tau_{m j_{m}} t_{\mathrm{j}}
$$

with extension by linearity over a suitable domain. Specifically, in the notation (2.1),

$$
\begin{aligned}
& \mathscr{D}\left(T_{m}^{\dagger}\right)=\left\{x: \Sigma_{\mathrm{j}} \tau_{m j_{m}}^{2}\left|\xi_{\mathrm{j}}\right|^{2}<\infty\right\}, \\
& T_{m}^{\dagger} x=\Sigma_{\mathrm{j}} \tau_{m j_{m}} \xi_{\mathrm{j}} t_{\mathrm{j}}, \quad x \in \mathscr{D}\left(T_{m}^{\dagger}\right) .
\end{aligned}
$$

An equivalent construction is given by Prugovečki [13, pp. 303-304], and it is readily verified that $V_{m n}^{\dagger}$ and $T_{m}^{\dagger}$ are selfadjoint, the $T_{m}^{\dagger}$ being strongly positive (1.2) and hence boundedly invertible.

Next we consider the determinant $\Delta(\omega)$ with $(m, n)$ th entry given by

$$
\Delta_{0 n}(\omega)=\omega_{n}, \quad \Delta_{m 0}(\omega)=-T_{m}^{\dagger}, \quad \Delta_{m n}(\omega)=V_{m n}^{\dagger}, \quad 0 \leqslant m, n \leqslant k,
$$

where $\omega=\left(\omega_{0}, \ldots, \omega_{k}\right) \in \mathbf{R}^{k+1}$. In the notation (1.5), $\Delta=\Delta\left(\alpha_{0}, \ldots, \alpha_{k}\right)$. Observe that operators from different rows of $\Delta(\omega)$ commute, so $\Delta(\omega)$ is defined unambiguously, on

$$
\mathscr{D}=\bigcap_{m=1}^{k} \mathscr{D}\left(T_{m}^{\dagger}\right)
$$


which is easily shown to be dense in $H$. The cofactors of $\omega_{n}=\Delta_{0 n}(\omega)$ in $\Delta(\omega)$ then define operators $\Delta_{n}$ on $\mathscr{D}$ : explicitly

$$
\Delta(\omega)=\sum_{n=0}^{k} \alpha_{n} \Delta_{n}, \quad \Delta_{n}=\sum_{m=1}^{k} T_{m}^{\dagger} \Delta_{0 m n}, \quad n=1, \ldots, k,
$$

where $\Delta_{0 m n}$ is the cofactor of $V_{m n}^{\dagger}$ in the expansion of $\Delta_{0}=\operatorname{det}\left[V_{m n}^{\dagger}\right]$. Note that the commutativity mentioned above forces each $\Delta_{n}$ to be symmetric.

Actually $\Delta_{0}$ can and will be defined on all of $H$, as a bounded selfadjoint operator. Similarly we define bounded selfadjoint operators $\Delta_{0 m n}$ on $H$ by means of the above cofactors, $1 \leqslant m, n \leqslant k$. Observe that

$$
\Sigma=\sum_{n=1}^{k} \alpha_{n} \Delta_{n}=\sum_{m=1}^{k} T_{m}^{\dagger} \sum_{n=1}^{k} \alpha_{n} \Delta_{0 m n}
$$

Our left definiteness assumption (1.4) guarantees

$$
\left(x^{\otimes}, \sum_{n=1}^{k} \alpha_{n} \Delta_{0 m n} x^{\otimes}\right) \geqslant 2 \delta\left\|x^{\otimes}\right\|^{2}, \quad m=1, \ldots, k,
$$

- see (1.3) - and a result of the author [2] extends this to all of $H$. Thus the following lemma and the (strong) positivity of the $T_{m}$ allow us to conclude that $\Sigma$ is strongly positive on $\mathscr{D}$.

LeMma 2.1. If $A_{l}$ are selfadjoint on $H_{l}, l=1,2$, with $A_{2}$ bounded, $\left(y, A_{2} y\right) \geqslant$ $\alpha_{2}\|y\|^{2} \forall y \in H_{2}$ and $\left(x, A_{1}^{\dagger} x\right) \geqslant \alpha_{1}\|x\|^{2} \forall x \in \mathscr{Q}\left(A_{1}^{\dagger}\right)$, then $\left(x, A_{1}^{\dagger} A_{2}^{\dagger} x\right) \geqslant \alpha_{1} \alpha_{2}\|x\|^{2}$ $\forall x \in \mathcal{D}\left(A_{1}^{\dagger}\right)$.

Proof. Apply [2] to the determinantal operator

$$
\otimes\left|\begin{array}{cc}
I_{1} & 0 \\
0 & A_{2}
\end{array}\right|=A_{2}^{\dagger}
$$

to give $\left(x, A_{2}^{\dagger} x\right) \geqslant \alpha_{2}\|x\|^{2}$. Then

$$
\begin{aligned}
\left(x, A_{1}^{\dagger} A_{2}^{\dagger} x\right) & =\left(\left(A_{2}^{\dagger}\right)^{1 / 2} x, A_{1}^{\dagger}\left(A_{2}^{\dagger}\right)^{1 / 2} x\right) \\
& \geqslant \alpha_{1}\left\|\left(A_{2}^{\dagger}\right)^{1 / 2} x\right\|^{2} \geqslant \alpha_{1} \alpha_{2}\|x\|^{2} \text {. Q.E.D. }
\end{aligned}
$$

3. Transformation of the eigenvalues. Continuity considerations show that (1.4) holds for perturbations of $\boldsymbol{\alpha} \in \mathbf{R}^{k}$ and $\delta>0$. More precisely, there exist linearly independent vectors $\boldsymbol{\alpha}^{m}=\left(\alpha_{1}^{m}, \ldots, \alpha_{k}^{m}\right), m=1, \ldots, k$, such that

$$
\sum_{n=1}^{k} \alpha_{n}^{m} \delta_{0 / n}(x)\left\|x_{l}\right\|^{2} \geqslant \delta\left\|x_{1}\right\|^{2} \cdots\left\|x_{k}\right\|^{2}, \quad 1 \leqslant l, m \leqslant k .
$$

We now introduce the eigenvalue transformation given by

$$
\lambda_{m}^{\prime}=\sum_{n=1}^{k} \alpha_{n}^{m} \lambda_{n}
$$


and we define $\theta_{n l}$ and $\theta$ by

$$
\lambda_{n}=\sum_{l=1}^{k} \theta_{n l} \lambda_{l}^{\prime}, \quad \theta=\operatorname{det}\left[\theta_{n l}\right]
$$

- we order the $\lambda_{m}^{\prime}$ so that $\theta>0$. Evidently (*) becomes

$$
T_{m} x_{m}=\sum_{l=1}^{k} \lambda_{l}^{\prime} \sum_{n=1}^{k} \theta_{n l} V_{m n} x_{m}, \quad 0 \neq x_{m} \in H_{m}, m=1, \ldots, k,
$$

so the transformed cofactors, constructed as in $\S 2$, obey

$$
\begin{gathered}
\Delta_{0 l m}^{\prime}=\theta \sum_{n=1}^{k} \alpha_{n}^{m} \Delta_{0 l n}, \\
\Delta_{0}^{\prime}=\theta \Delta_{0}, \quad \Delta_{n}^{\prime}=\sum_{m=1}^{k} T_{m}^{\dagger} \Delta_{0 m n}^{\prime}, \quad 1 \leqslant l, m, n \leqslant k .
\end{gathered}
$$

It follows from (3.1) and [2] that each $\Delta_{0 / m}^{\prime} \gg 0$ on $H$ and so from Lemma 2.1 that each $\Delta_{n}^{\prime} \gg 0$ on $\mathscr{D}, n=1, \ldots, k$. From now on, we shall drop the primes, and we summarise our conclusions so far.

THEOREM 3.1. After a possible nonsingular linear transformation of the eigenvalues, we may assume that

$$
\Delta_{0 m n} \geqslant \delta I \text { on } H, \quad 1 \leqslant m, n \leqslant k,
$$

and that each $\Delta_{n}$ is strongly positive on $\mathscr{D}, n=1, \ldots, k$.

So far, we have defined the $\Delta_{n}$ only on $\mathscr{D}, n=1, \ldots, k$, but we shall now choose a strongly positive selfadjoint extension $\hat{\Delta}_{n}$. That this is possible follows from Theorem 3.1 and [8, Theorem XII.5.2]. In fact Theorem 5.1 and [16, Theorem (4.6)] show that $\hat{\Delta}_{n}$ is unique, so $\hat{\Delta}_{n}=\Delta_{n}^{*}$.

LEMMA 3.2. $\Delta_{n}(\mathscr{D})$ and $\hat{\Delta}_{n}^{1 / 2}(\mathscr{D})$ are dense in $H$.

Proof. If, for all $x \in \mathscr{D}, 0=\left(y, \Delta_{n} x\right)$ then $y \in \mathscr{D}\left(\Delta_{n}^{*}\right)$ and $\Delta_{n}^{*} y=0$ since $\mathscr{D}$ is dense in $H$. From the above remark, $\hat{\Delta}_{n} y=0$ and $\hat{\Delta}_{n}$ is strongly positive, so $\hat{\Delta}_{n}^{-1}$ is bounded, whence $y=0$.

It follows that $\Delta_{n}(\mathscr{D})$ is dense in $H$ and therefore $\hat{\Delta}_{n}^{1 / 2} \mathscr{D}$ is dense in $\Re\left(\hat{\Delta}_{n}^{1 / 2}\right)$. But $\hat{\Delta}_{n}^{-1 / 2}$ is bounded, so $R\left(\Delta_{n}^{-1 / 2}\right)=H$. Q.E.D.

Since $\hat{\Delta}_{n}$ is strongly positive, $\hat{\Delta}_{n}^{-1}$ is bounded, and in fact more is true.

THEOREM 3.3. $\hat{\Delta}_{n}^{-1}$ is compact.

Proof. Consider the operator

$$
E=\sum_{m=1}^{k} T_{m}^{\dagger}, \quad \mathscr{D}(E)=\mathscr{D} .
$$


It is easily verified that $E$ is selfadjoint with eigenvectors $t_{\mathbf{j}}$ and corresponding eigenvalues

$$
\tau_{\mathbf{j}}=\sum_{m=1}^{k} \tau_{m j_{m}} .
$$

In particular, $E$ has compact inverse and so $E^{-1 / 2}$ is compact.

From (3.2) and Lemma 2.1,

$$
\Delta_{n}-\delta E=\sum_{m=1}^{k} T_{m}^{\dagger}\left(\Delta_{0 m n}-\delta I\right) \geqslant 0
$$

on $\mathscr{D}$. Now let $x \in \mathscr{D}$ and $y=\hat{\Delta}_{n}^{1 / 2} x$. Then

$$
\frac{\left\|E^{1 / 2} \hat{\Delta}_{n}^{-1 / 2} y\right\|^{2}}{\|y\|^{2}}=\frac{(x, E x)}{\left(x, \Delta_{n} x\right)} \leqslant \delta^{-1} .
$$

It follows that $E^{1 / 2} \hat{\Delta}_{n}^{-1 / 2}$ is bounded on $\hat{\Delta}_{n}^{1 / 2}(\mathscr{D})$, and hence has a bounded extension $B$ to all of $H$ by Lemma 3.2.

Thus

$$
\hat{\Delta}_{n}^{-1 / 2}=E^{-1 / 2} B
$$

is compact, and hence so is $\hat{\Delta}_{n}^{-1}$. Q.E.D.

We are now in a position to construct the compact operators we shall use for our subsequent analysis.

CoROllaRY 3.4. $B_{n}=\hat{\Delta}_{n}^{-1} \Delta_{0}$ is compact, and $B_{n}^{\prime}=\hat{\Delta}_{n}^{-1 / 2} \Delta_{0} \hat{\Delta}_{n}^{-1 / 2}$ is compact and selfadjoint, on $H$ for $n=1, \ldots, k$.

4. Limiting procedures. We shall find it convenient at various points to employ a limiting procedure which is related to, but different from, that used by Browne [6] and Sleeman [14]. We define bounded selfadjoint operators $T_{m}^{N}$ and $\Delta_{n}^{N}$ on $H$ by

$$
\begin{aligned}
& T_{m}^{N} t_{\mathbf{j}}=\tau_{m j} t_{\mathbf{j}} \quad \text { if } 1 \leqslant j_{m} \leqslant N, \\
& T_{m}^{N} t_{\mathbf{j}}=\tau_{m N} t_{\mathbf{j}} \quad \text { if } j_{m} \geqslant N,
\end{aligned}
$$

and

$$
\Delta_{n}^{N}=\sum_{m=1}^{k} T_{m}^{N} \Delta_{0 m n}
$$

Note that symmetry of $\Delta_{n}^{N}$ follows as for that of $\Delta_{n}$. It is evident that $T_{m}^{N} \geqslant \tau_{m 1} I$, so if we set $\tau_{1}=\sum_{m=1}^{k} \tau_{m 1}$-cf. (3.3) - and use Lemma 2.1 and (3.2) then we have

$$
\Delta_{n}^{N} \geqslant \delta \tau_{1} I, \quad n=1, \ldots, k .
$$

It follows that $T_{m}^{N}$ and $\Delta_{n}^{N}$ are invertible with

$$
\left\|\left(T_{m}^{N}\right)^{-1}\right\| \leqslant \tau_{m 1}^{-1}, \quad\left\|\left(\Delta_{n}^{N}\right)^{-1}\right\| \leqslant\left(\delta \tau_{1}\right)^{-1}
$$

and we set

$$
B_{n}^{N}=\left(\Delta_{n}^{N}\right)^{-1} \Delta_{0}
$$


LEMMA 4.1. $\left(T_{m}^{N}\right)^{-1} \rightarrow\left(T_{m}^{\dagger}\right)^{-1}$ and $B_{n}^{N} \rightarrow B_{n}$ as $N \rightarrow \infty$ strongly on $H$.

Proof. Let $x \in \mathscr{D}\left(T_{m}^{\dagger}\right)$, so $T_{m}^{N} x \rightarrow T_{m}^{\dagger} x$. Thus if $y=T_{m}^{\dagger} x$, then

$$
\left\|\left(T_{m}^{N}\right)^{-1} y-\left(T_{m}^{\dagger}\right)^{-1} y\right\|=\left\|\left(T_{m}^{N}\right)^{-1}\left(T_{m}^{\dagger}-T_{m}^{N}\right) x\right\| \rightarrow 0,
$$

by (4.1). The first claim then follows because $T_{m}^{\dagger}$ is onto.

Likewise, if $u \in \mathscr{D}$ and $v=\Delta_{n} u$ then

$$
\left\|\left(\Delta_{n}^{N}\right)^{-1} v-\Delta_{n}^{-1} v\right\|=\left\|\left(\Delta_{n}^{N}\right)^{-1}\left(\Delta_{n}-\Delta_{n}^{N}\right) u\right\| \rightarrow 0 .
$$

It follows from Lemma 3.2 that such $v$ are dense in $H$. Since $\left(\Delta_{n}^{N}\right)^{-1}$ is uniformly bounded in $N(4.1),\left(\Delta_{n}^{N}\right)^{-1} \rightarrow \hat{\Delta}_{n}^{-1}$, strongly on $H$. The required result is now immediate. Q.E.D.

We are now ready for the first application of these ideas to (*).

THEOREM 4.2. The operators $B_{n}$ are pairwise commutative.

Proof. We first consider the system of equations

$$
T_{m}^{N} z_{0}=\sum_{n=1}^{k} V_{m n} z_{n}, \quad m=1, \ldots, k .
$$

It follows from [11, Theorem 2] and the fact that $\left(\Delta_{l}^{N}\right)^{-1}$ is bounded that (4.3) is soluble for $z_{0}, \ldots, z_{l-1}, z_{l+1}, \ldots, z_{k}$ in terms of $z_{l}$. Further [11, equation (3.2)] gives

$$
\Delta_{m}^{N} z_{l}=\Delta_{l}^{N} z_{m}, \quad 0 \leqslant l, m \leqslant k,
$$

where we have written $\Delta_{0}^{N}=\Delta_{0}$ for convenience.

In particular, then,

$$
z_{0}=\left(\Delta_{l}^{N}\right)^{-1} \Delta_{0} z_{l}=\left(\Delta_{m}^{N}\right)^{-1} \Delta_{0} z_{m}=\left(\Delta_{m}^{N}\right)^{-1} \Delta_{0}\left(\Delta_{l}^{N}\right)^{-1} \Delta_{m}^{N} z_{l} .
$$

Setting $z_{l}=B_{m}^{N} z$, we then find

$$
B_{l}^{N} B_{m}^{N}=B_{m}^{N} B_{l}^{N}, \quad 1 \leqslant l, m \leqslant k .
$$

Now (4.1) shows that the $B_{n}^{N}$ are uniformly bounded in $N$, so Lemma 4.1 yields

$$
B_{l} B_{m}=B_{m} B_{l}
$$

as required. Q.E.D.

5. Analysis of eigenvectors. We shall now analyse the compact operators $B_{m}$ and $B_{m}^{\prime}$ introduced in Corollary 3.4. Let $\beta_{m j}, j=1,2, \ldots$, be the distinct nonzero eigenvalues of $B_{m}^{\prime}$ with corresponding eigenspaces $F_{m j}^{\prime}$. Now if $f^{\prime} \in F_{m j}^{\prime}$ and $f=$ $\hat{\Delta}_{m}^{-1 / 2} f^{\prime}$, then

$$
B_{m} f=\beta_{m j} f .
$$

Conversely, if (5.1) holds then $f \in \mathscr{D}\left(\hat{\Delta}_{m}\right)$ so $f^{\prime}=\hat{\Delta}_{m}^{1 / 2} f \in F_{m j}^{\prime}$. It follows that $B_{m}$ has the same nonzero eigenvalues $\beta_{m j}$ with corresponding finite dimensional eigenspaces $F_{m j}=\hat{\Delta}_{m}^{-1 / 2} F_{m j}^{\prime}$, isomorphic to $F_{m j}^{\prime}$.

It will be convenient for us to have the notation $K$ for $\operatorname{Ker} \Delta_{0}$ and to write

$$
K_{m}=\hat{\Delta}_{m}^{-1}\left(K^{\perp}\right)
$$

so $x \in K_{m}$ iff $x \in \mathscr{D}\left(\hat{\Delta}_{m}\right)$ and $\Delta_{0} y=0 \Rightarrow\left(y, \hat{\Delta}_{m} x\right)=0$. 
LEMMA 5.1. The $F_{m j}, j=1,2, \ldots$, form a fundamental set in $K_{m}$.

Proof. First, if (5.1) holds then $f \in \mathcal{D}\left(\hat{\Delta}_{m}\right)$ and

$$
\Delta_{0} y=0 \Rightarrow\left(y, \hat{\Delta}_{m} f\right)=\beta_{m j}^{-1}\left(y, \Delta_{0} f\right)=\beta_{m j}^{-1}\left(\Delta_{0} y, f\right)=0 .
$$

Thus $f \in K_{m}$ so indeed $F_{m j} \subseteq K_{m}$.

Now let $z \in K_{m}$ and $x=\hat{\Delta}_{m}^{1 / 2} z$. Suppose $e \in \operatorname{Ker} B_{m}^{\prime}$, so

$$
\hat{\Delta}_{m}^{-1 / 2} e \in K \text {. }
$$

But $x \in \hat{\Delta}_{m}^{-1 / 2}\left(K^{\perp}\right)$ so $(x, e)=0$ and $x \in\left(\operatorname{Ker} B_{m}^{\prime}\right)^{\perp}$. Thus $x$ belongs to the closure of the span of the $F_{m j}^{\prime}, j=1,2, \ldots$, i.e. the $F_{m j}^{\prime}$ are fundamental in $\hat{\Delta}_{m}^{-1 / 2}\left(K^{\perp}\right)$. The result follows directly. Q.E.D.

We are now ready to generalise some work of Atkinson [1, §3.6] to our infinite dimensional situation as follows.

THEOREM 5.2. Let $1 \leqslant l \leqslant k$. Then the $B_{n}, n=1, \ldots, k$, possess a fundamental set of common eigenvectors in $K_{l}$.

Proof. From Theorem 4.2 we have

$$
B_{m} F_{n j} \subseteq F_{n j}, \quad 1 \leqslant m, n \leqslant k .
$$

If $\operatorname{dim} F_{1 j}=1$ for each $j$ then we choose nonzero $f_{1 j} \in F_{1 j}$ and conclude that the $f_{1 j}$ form a common set of eigenvectors for the $B_{n}$ by (5.3). Fundamentality then follows from Lemma 5.1.

If $B_{1}$ has multiple eigenvalues, then we proceed as follows. Let $B_{2 j}$ be the restriction of $B_{2}$ to its invariant (5.3) subspace $F_{1 j}, j=1,2, \ldots$ Observe that if $B_{2 j} f=0$ then $f \in F_{1 j} \cap K$ so $B_{1} f=0$. Thus $f=0$ and so $B_{2 j}$ is nonsingular. By virtue of the correspondence between eigenvectors of $B_{2}$ and $B_{2}^{\prime}$, we see that $F_{1 j}$ is spanned by eigenvectors of $B_{2}$ with nonzero corresponding eigenvalues. Consequently we may construct subspaces

$$
F_{\mathbf{j}}^{(2)}=F_{1 j_{1}} \cap F_{2 j_{2}}, \quad \mathbf{j}=\left(j_{1}, j_{2}\right),
$$

of eigenvectors common to $B_{1}$ and $B_{2}$ for each positive integer 2-vector $\mathbf{j}$. From Lemma 5.1, the $F_{\mathrm{j}}^{(2)}$ are fundamental in $K_{1}$ and $K_{2}$.

Note that (5.3) shows that $F_{j}^{(2)}$ is invariant for each $B_{n}, n=1, \ldots, k$. Thus we can repeat the process, restricting $B_{3}$ to $F_{\mathbf{j}}^{(2)}$ and generating invariant subspaces

$$
F_{\mathbf{j}}^{(3)}=F_{1 j_{1}} \cap F_{2 j_{2}} \cap F_{3 j_{3}}, \quad \mathbf{j}=\left(j_{1}, j_{2}, j_{3}\right),
$$

of eigenvectors common to $B_{1}, B_{2}$ and $B_{3}$, and so on. At the final stage the $F_{j}^{(k)}$ consist of common eigenvectors for the $B_{n}, n=1, \ldots, k$, for each positive integer $k$-vector $\mathbf{j}$. The set of all such $F_{\mathbf{j}}^{(k)}$ is fundamental in $K_{n}, n=1, \ldots, k$. If $\operatorname{dim} F_{\mathbf{j}}^{(k)}=1$ for each $\mathbf{j}$, then we simply pick any nonzero $f_{\mathbf{j}} \in F_{\mathbf{j}}^{(k)}$ to complete the construction. If $\operatorname{dim} F_{\mathbf{j}}^{(k)}>1$ for any integer $k$-vector $\mathbf{j}$ then we may choose any basis for $F_{\mathbf{j}}^{(k)}$. Q.E.D.

This result may be given more structure at the cost of a more complicated construction. We define $\mathscr{D}_{l}$ as $\mathscr{D}\left(\hat{\Delta}^{1} /{ }^{2}\right)$ under the inner product $[,]_{l}$ given by

$$
[x, y]_{l}=\left[\hat{\Delta}^{1} /{ }^{2} x, \hat{\Delta}^{1} /{ }^{2} y\right], \quad l=1, \ldots, k \text {. }
$$


It can be shown $[3, \S 2.4 .2]$ that $\mathscr{D}_{l}$ is a Hilbert space, and in fact is the completion of Q $)$ under $[,]_{l}$. (This follows because

$$
[x, y]_{l}=\left(x, \hat{\Delta}_{l} y\right) \text { if } x \in \mathscr{Q}_{l}, y \in \mathscr{Q}\left(\hat{\Delta}_{l}\right) \text {. }
$$

Thus if $x$ is $\mathscr{D}_{l}$-orthogonal to $\mathscr{Q} \subseteq \mathscr{Q}\left(\hat{\Delta}_{l}\right), x$ must be $H$-orthogonal to $\hat{\Delta}_{l}(\mathscr{D})=\Delta_{l}(\mathscr{Q})$. Since $\Delta_{l}(\bigotimes)$ is dense in $H$ by Lemma 3.2, $x$ must vanish.)

LEMMA 5.3. $K \cap \mathscr{D}_{l}$ is closed in $\mathscr{D}_{l}$, where $K=\operatorname{Ker} \Delta_{0}$.

Proof. Suppose $x_{j} \in K \cap \mathscr{Q}\left(\hat{\Delta}^{1 / 2}\right)$ with $x_{l} \rightarrow x$ in $\mathscr{D}_{l}$. Then $x_{j} \rightarrow x$ in $H$, so $0=\Delta_{0} x_{j} \rightarrow \Delta_{0} x$. Q.E.D.

This enables us to define

$$
\mathscr{K}_{l}=\left(K \cap \mathscr{D}_{l}\right)^{\perp}, \quad \mathscr{K}_{l} \oplus\left(K \cap \mathscr{D}_{l}\right)=\mathscr{D}_{l},
$$

OD - orthogonality being understood. It is easily verified, using (5.4), that $\mathscr{K}_{l}$ and $K_{l}$ (5.2) are connected by the relation

$$
K_{l}=\mathscr{K}_{l} \cap \mathscr{D}\left(\hat{\Delta}_{l}\right)
$$

We can now recast our earlier work as follows.

Corollary 5.4. A set of common eigenvectors for the $B_{n}, n=1, \ldots, k$, may be chosen to form a complete orthogonal basis of all the $\mathcal{K}_{m}, m=1, \ldots, k$, and by normalisation it may also be chosen orthonormal in any individual $\mathcal{K}_{l}$.

Proof. Let $f_{\mathbf{i}} \in F_{\mathbf{i}}^{(k)}$ and $f_{\mathbf{j}} \in F_{\mathbf{j}}^{(k)}$ be eigenvectors constructed as in Theorem 5.2. Then

$$
B_{m} f_{\mathbf{i}}=\alpha_{m \mathbf{i}} f_{\mathbf{i}}, \quad B_{m} f_{\mathbf{j}}=\alpha_{m \mathbf{j}} f_{\mathbf{j}}, \quad m=1, \ldots, k,
$$

say. Thus

$$
\left[f_{\mathbf{i}}, B_{m} f_{\mathbf{j}}\right]_{m}=\alpha_{m \mathbf{i}}\left[f_{\mathbf{i}}, f_{\mathbf{j}}\right]_{m}=\alpha_{m \mathbf{j}}\left[f_{\mathbf{i}}, f_{\mathbf{j}}\right]_{m}, \quad m=1, \ldots, k .
$$

Now if $\mathbf{i} \neq \mathbf{j}$ then $\boldsymbol{\alpha}_{m \mathbf{i}} \neq \boldsymbol{\alpha}_{m \mathbf{j}}$ for some $m$, so $f_{\mathbf{i}}$ and $f_{\mathbf{j}}$ are $\mathscr{Q}_{m}$-orthogonal.

Linear independence of eigenvectors within the $F_{j}^{(k)}$ was guaranteed by construction, so we simply make $\mathscr{D}_{\text {l }}$-orthonormal eigenvectors by the Gram-Schmidt process. Suppose, then, that $\mathbf{i}=\mathbf{j}$ but $f_{\mathbf{i}} \neq f_{\mathbf{j}}$ so $\left[f_{\mathbf{i}}, f_{\mathbf{j}}\right]_{l}=0$. From (5.6),

$$
\Delta_{0} f_{\mathbf{j}}=\alpha_{m \mathbf{j}} \Delta_{m} f_{\mathbf{j}}=\alpha_{l \mathbf{j}} \Delta_{l} f_{\mathbf{j}}
$$

so

$$
\begin{aligned}
{\left[f_{\mathbf{i}}, f_{\mathbf{j}}\right]_{m} } & =\left(f_{\mathbf{i}}, \Delta_{m} f_{\mathbf{j}}\right) \quad \text { by }(5.4) \\
& =\boldsymbol{\alpha}_{l \mathbf{j}} \boldsymbol{\alpha}_{m \mathbf{j}}^{-1}\left(f_{\mathbf{i}}, \Delta_{l} f_{\mathbf{j}}\right)=\left[f_{\mathbf{i}}, f_{\mathbf{j}}\right]_{l}=0 .
\end{aligned}
$$

Thus the $f_{\mathbf{j}}$ are indeed $\mathscr{D}_{m}$-orthogonal for each $m$. Q.E.D.

6. A spectral theory for $(*)$. We shall now relate the above work to the original problem. First, $(*)$ is equivalent to

$$
T_{m}^{\dagger} x=\sum_{n=1}^{k} \lambda_{n} V_{m n}^{\dagger} x, \quad 0 \neq x \in \mathscr{D}, m=1, \ldots, k,
$$


in the sense that [6, Lemma 5]

$$
\bigotimes_{m=1}^{k} \operatorname{Ker}\left(T_{m}-\sum_{n=1}^{k} \lambda_{n} V_{m n}\right)=\bigcap_{m=1}^{k} \operatorname{Ker}\left(T_{m}^{\dagger}-\sum_{n=1}^{k} \lambda_{n} V_{m n}^{\dagger}\right) .
$$

Thus if $(*)$ generates $j$ linearly independent tensors $x^{\otimes}(1.3)$ for some $\left(\lambda_{1}, \ldots, \lambda_{k}\right)$ then so does (6.1). Conversely, if $j$ linearly independent elements $x$ satisfy (6.1), then so do $j$ linearly independent elements of the form $x^{\otimes}$ where the corresponding $x_{m}$ satisfy $(*)$.

We shall next establish another equivalent formulation.

THEOREM 6.1. (*) is equivalent to

$$
x=\lambda_{n} B_{n} x, \quad 0 \neq x \in H, n=1, \ldots, k .
$$

Proof. We return to the associated system (4.3) which we shall solve for $z_{0}, z_{2}, \ldots, z_{k}$ given $z_{1}=\prod_{l=2}^{k} B_{l}^{N} z$ where $z \in H$. By virtue of Theorem 4.2, we have

$$
z_{0}=\prod_{l=1}^{k} B_{l}^{N} z, \quad z_{n}=\prod_{\substack{l=1 \\ l \neq n}}^{k} B_{l}^{N} z, \quad n=1, \ldots, k
$$

from (4.4). Thus (4.3) yields

$$
\prod_{l=1}^{k} B_{l}^{N}=\left(T_{m}^{N}\right)^{-1} \sum_{n=1}^{k} V_{m n}^{\dagger} \prod_{\substack{l=1 \\ l \neq n}}^{k} B_{l}^{N}, \quad m=1, \ldots, k,
$$

since $z$ was an arbitrary element of $H$.

Making use of (4.1) and Lemma 4.1 we may pass to the limit in (6.3)-applied to $\lambda_{1} \cdots \lambda_{k} x$ with $x \in H$ - to obtain

$$
\prod_{l=1}^{k} \lambda_{l} B_{l} x=\left(T_{m}^{\dagger}\right)^{-1} \sum_{n=1}^{k} V_{m n}^{\dagger} \lambda_{n} \prod_{\substack{l=1 \\ l \neq n}}^{k} \lambda_{l} B_{l} x, \quad m=1, \ldots, k
$$

Now if we suppose that $x$ satisfies (6.2), then we have

$$
x=\left(T_{m}^{\dagger}\right)^{-1} \sum_{n=1}^{k} \lambda_{n} V_{m n}^{\dagger} x, \quad m=1, \ldots, k,
$$

which obviously implies (6.1), and hence (*).

Conversely, if $(*)$ holds then so does $(6.1)$ and therefore

$$
\begin{aligned}
\Delta_{l} x & =\sum_{m=1}^{k} \Delta_{0 m l} \sum_{n=1}^{k} \lambda_{n} V_{m n}^{\dagger} x \\
& =\sum_{n=1}^{k} \lambda_{n} \delta_{l n} \Delta_{0} x=\lambda_{l} \Delta_{0} x, \quad l=1, \ldots, k,
\end{aligned}
$$

by a standard identity, e.g. [11, equation (2.2)]. Thus (6.2) holds as required. Q.E.D.

Now we are in a position to draw our main conclusions. Notice that (6.2) is a simultaneous eigenvector problem for the $B_{n}$, where the corresponding eigenvalues 
$\lambda_{n}^{-1}$ are nonzero. This is precisely the problem to which $\S 5$ applies, and accordingly we have the following result, in terms of the subspaces $K_{l} \subseteq H$ (5.2) and $\mathcal{K}_{l} \subseteq \mathscr{D}_{l}$ (5.5).

THEOREM 6.2. The eigenvalues $\lambda_{n}$ of $(*)$ are the nonzero eigenvalues of $B_{n}$, and are thus real with finite multiplicity and with no finite accumulation points. The eigenvectors of $(*)$ generate tensors $x^{\otimes}$ (1.3) forming a set $S$ of common eigenvectors of the $B_{n}$ and $S$ is fundamental in $K_{l}, l=1, \ldots, k$. Finally, complete orthonormal bases for the $\mathcal{K}_{l}, l=1, \ldots, k$, may be chosen from $S$.

\section{REFERENCES}

1. F. V. Atkinson, Multiparameter eigenvalue problems, Vol. 1, Academic Press, New York and London, 1972.

2. P. A. Binding, Another positivity result for determinantal operators, Proc. Roy. Soc. Edinburgh 86A (1980), 333-337.

3.

4. P. J. Browne, A multiparameter eigenvalue problem, J. Math. Anal. Appl. 38 (1972), 553-568.

5. __ Multiparameter spectral theory, Indiana Univ. Math. J. 24 (1974), 249-257.

6. __ Abstract multiparameter theory. I, J. Math. Anal. Appl. 60 (1977), 259-273.

7. __ Abstract multiparameter theory. II, J. Math. Anal. Appl. 60 (1977), 274-279.

8. N. Dunford and J. T. Schwartz, Linear operators. Part II, Interscience, New York, 1963.

9. M. Faierman, The completeness and expansion theorems associated with the multiparameter eigenvalue problem in ordinary differential equations, J. Differential Equations 5 (1969), 197-213.

10. A. Källström and B. D. Sleeman, A left definite multiparameter eigenvalue problem in ordinary differential equations, Proc. Roy. Soc. Edinburgh Sect. A 74 (1976), 145-155.

11. ___ Solvability of a linear operator system, J. Math. Anal. Appl. 55 (1976), 785-793.

12. __ Multiparameter spectral theory, Ark. Mat. 15 (1977), 93-99.

13. E. Prugovečki, Quantum mechanics in Hilbert space, Academic Press, New York and London, 1971.

14. B. D. Sleeman, Multiparameter spectral theory in Hilbert space, J. Math. Anal. Appl. 65 (1978), $511-530$.

15. L. Turyn, Sturm-Liouville problems with several parameters, J. Differential Equations 38 (1980), 239-259.

16. H. Volkmer, On multiparameter theory, J. Math. Anal. Appl. 86 (1982), 44-53.

Department of Mathematics and Statistics, University of Calgary, Calgary, Alberta, CANADA T2N $1 \mathrm{~N} 4$ 